\title{
Effects of antidepressants on the conformation of phospholipid headgroups studied by solid-state NMR
}

\author{
Jose S. Santos, ${ }^{1 \dagger}$ Dong-Kuk Lee ${ }^{1,2}$ and Ayyalusamy Ramamoorthy ${ }^{1,2,3 *}$ \\ 1 Biophysics Research Division, University of Michigan, Ann Arbor, Michigan 48109-1055, USA \\ ${ }^{2}$ Department of Chemistry, University of Michigan, Ann Arbor, Michigan 48109-1055, USA \\ ${ }^{3}$ Macromolecular Science and Engineering, University of Michigan, Ann Arbor, Michigan 48109-1055, USA
}

Received 10 June 2003; Revised 25 August 2003; Accepted 1 September 2003

\begin{abstract}
The effect of tricyclic antidepressants (TCA) on phospholipid bilayer structure and dynamics was studied to provide insight into the mechanism of TCA-induced intracellular accumulation of lipids (known as lipidosis). Specifically we asked if the lipid-TCA interaction was TCA or lipid specific and if such physical interactions could contribute to lipidosis. These interactions were probed in multilamellar vesicles and mechanically oriented bilayers of mixed phosphatidylcholine-phosphatidylglycerol (PC-PG) phospholipids using ${ }^{31} \mathrm{P}$ and ${ }^{14} \mathrm{~N}$ solid-state NMR techniques. Changes in bilayer architecture in the presence of TCAs were observed to be dependent on the TCA's effective charge and steric constraints. The results further show that desipramine and imipramine evoke distinguishable changes on the membrane surface, particularly on the headgroup order, conformation and dynamics of phospholipids. Desipramine increases the disorder of the choline site at the phosphatidylcholine headgroup while leaving the conformation and dynamics of the phosphate region largely unchanged. Incorporation of imipramine changes both lipid headgroup conformation and dynamics. Our results suggest that a correlation between TCA-induced changes in bilayer architecture and the ability of these compounds to induce lipidosis is, however, not straightforward as imipramine was shown to induce more dramatic changes in bilayer conformation and dynamics than desipramine. The use of ${ }^{14} \mathrm{~N}$ as a probe was instrumental in arriving at the presented conclusions. Copyright $\odot 2004$ John Wiley \& Sons, Ltd.
\end{abstract}

KEYWORDS: NMR; ${ }^{14}$ N NMR; ${ }^{31}$ P NMR; desipramine; imipramine; iminodibenzyl; phospholipid; antidepressant; tricyclic antidepressants

\section{INTRODUCTION}

The class of tricyclic antidepressants, which includes imipramine and its metabolized product desipramine (Fig. 1), is known to interact strongly with the lipid bilayer. ${ }^{1-5}$ The 'amine hypothesis' suggests that the pharmacological effect of these drugs is to block the re-uptake of neurotransmitters at the nerve synapses. ${ }^{6}$ It is also known that the incorporation of these compounds into lipid bilayers leads to intracellular accumulation of various phospholipids, a process known as lipidosis. ${ }^{7-12}$ Tricyclic antidepressant (TCA)-induced lipidosis has been linked to the ability of these compounds to inhibit phospholipases which are responsible for phospholipid degradation. ${ }^{6,13,14}$ However, the mechanistic connection between TCA-lipid interaction and phospholipidosis remains puzzling. It is unclear whether a TCA interacts directly with the phospholipids and consequently

*Correspondence to: Ayyalusamy Ramamoorthy, Biophysics Research Division, University of Michigan, Ann Arbor, Michigan 48109-1055, USA. E-mail: ramamoor@umich.edu

${ }^{\dagger}$ Present address: Division of Biological Sciences, Section of

Neurobiology, University of California at San Diego, La Jolla,

California 92093-0366, USA.

Contract/grant sponsor: National Science Foundation.

Contract/grant sponsor: Petroleum Research Fund. renders the lipid unsuitable for degradation, or with the phospholipase thereby inactivating the enzyme. ${ }^{11}$ Since the process of sequestering peripheral membrane and cytosolic proteins to the membrane has been observed to be initially driven by an electrostatic interaction between the lipid headgroups and protein, ${ }^{15-18}$ it is plausible that the introduction of the charged TCA drug changes the electrostatic profile of the lipid bilayer and therefore may contribute to inhibit the phospholipase-membrane contact. In this conceptual frame, it is important to ask how the lipid bilayer structure and dynamics change at the atomic level upon the introduction of positively charged amphiphiles. Specifically, it would be valuable to test if there is a correlation between the different observed physiological effects of TCA homologues, such as desipramine and imipramine, and spectroscopically observable changes in the electrostatic profile of the membrane surface.

The introduction of any type of inclusion, here defined as any non-lipid molecule, into a lipid bilayer rearranges the number and type of interactions that stabilize the bilayer structure. Probing these types of interactions in a quantitative manner has proven difficult through spectroscopic methods. The work of Pasenkiewicz-Gierula and co-workers using molecular simulations of lipid bilayers to investigate 
A<smiles>c1ccc2c(c1)CCc1ccccc1N2</smiles><smiles>CNCCCN1c2ccccc2CCc2ccccc21</smiles><smiles>C=[N+](C)CCCN1c2ccccc2CCc2ccccc21</smiles>

Figure 1. Structures of TCA drugs: (A) iminodibenzyl; (B) desipramine; (C) imipramine.

these types of forces has recently produced a wealth of information. ${ }^{19,20}$ These studies showed that there are two types of short-distance interactions that are predicted to occur between phosphatidylcholine (PC) headgroups. These interactions are water-mediated hydrogen bonds and charge association (charge pairs), each of which can be inter- or intramolecular. ${ }^{19,21,22}$ Such interactions are responsible for the formation of an extended network among PC headgroups linking 98\% PC in a membrane. ${ }^{19}$ The sites of these interactions are the positively charged nitrogen atom in the choline headgroup, the negatively charged non-ester phosphate oxygens (see $\mathrm{O}_{13}$ in Fig. 2) and the carbonyl oxygens (see $\mathrm{O}_{22}$ and $\mathrm{O}_{32}$ in Fig. 2).

Solid-state NMR techniques on naturally abundant NMRactive nuclei of ${ }^{31} \mathrm{P}$ and ${ }^{14} \mathrm{~N}$ (100.0 and $99.63 \%$, respectively) are ideal to study these changes in the environment near the lipid headgroup in model lipid bilayers. Nitrogen-14 is a quadrupole nucleus that shares deuterium's sensitivity to changes in bilayer surface charge (the 'molecular voltmeter effect'). ${ }^{23}$ In spite of its high natural abundance and sensitivity to changes in electric field gradient, relatively few studies have made use of this nucleus. ${ }^{1,24-32}$ From the results of these studies, three important conclusions of particular relevance to this investigation can be drawn. First, the ${ }^{14} \mathrm{~N}$ nucleus appears to be more sensitive than deuterium since slight distortions of the tetrahedral symmetry upon addition of anionic amphiphiles result in large spectral changes. ${ }^{32}$ Second, the order of the choline headgroup is independent of the order of the acyl chains and the phosphate moiety, as shown by the observed concurrent $28 \%$ decrease in the ${ }^{14} \mathrm{~N}$ quadrupole splitting and $20 \%$ increase in ${ }^{31} \mathrm{P}$ chemical shift anisotropy (CSA) span upon addition of the local anesthetic tetracaine (at a lipid to tetracaine molar ratio of $2.6: 1) .{ }^{30}$ Finally, the choline headgroup is sensitive to desipramine, as evidenced by the $50 \%$ decrease in the lipid quadrupole splitting in the presence of the drug in a 1:2 mole ratio. ${ }^{1}$ Nitrogen-14 has an additional advantage in studying model lipid bilayers as it allows for the isolated study of PC since most other ${ }^{14} \mathrm{~N}$ nuclei (from proteins, other lipids or drugs) do not contribute to the ${ }^{14} \mathrm{~N}$ NMR signal due to large quadrupole coupling constants.

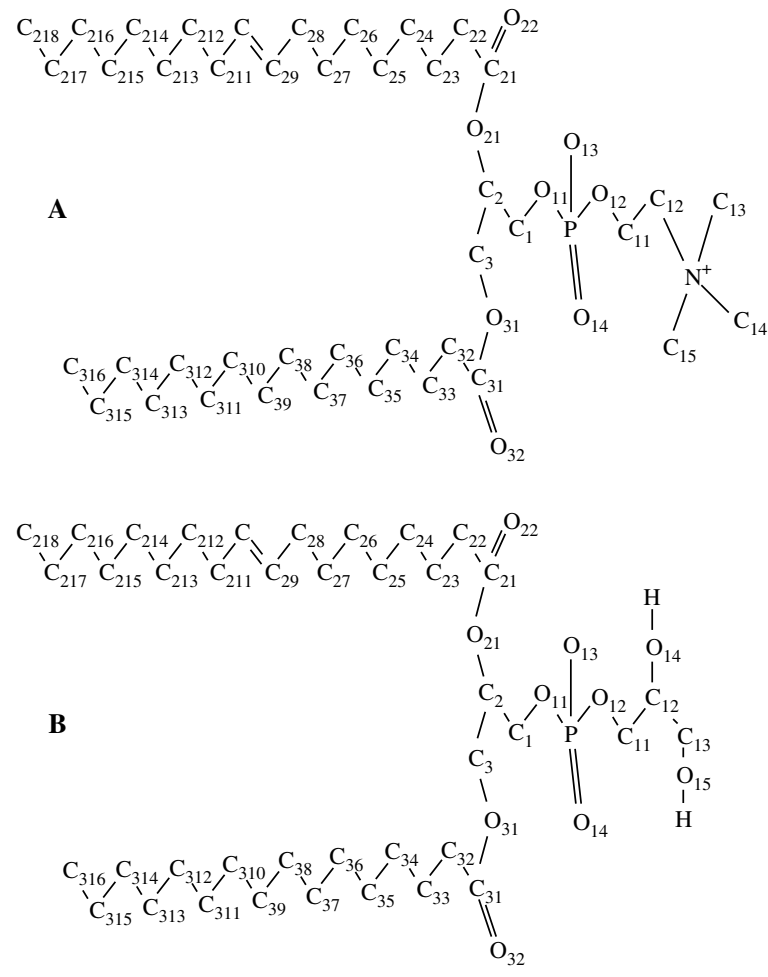

Figure 2. Structures of (A) 1-palmitoyl-2-oleoyl-sn-glycero3-phosphatidylcholine (POPC) and (B) 1-palmitoyl-2-oleoyl-snglycero-3-phosphatidylglycerol (POPG). The phospholipid atom numbering scheme is identical with that used by Pasenkiewicz-Gierula et al. ${ }^{19}$

In this study, the interaction of TCAs with mechanically oriented lipid bilayers and multilamellar lipid vesicles composed of a mixture of zwitterionic (PC) and anionic (phosphatidylglycerol) lipids was monitored by solid-state NMR techniques using as probes the ${ }^{31} \mathrm{P}$ and ${ }^{14} \mathrm{~N}$ NMR active nuclei located at the lipid headgroups. In general, it is shown that ${ }^{14} \mathrm{~N}$ NMR may be coupled to ${ }^{31} \mathrm{P}$ NMR techniques to distinguish between changes in lipid headgroup conformation and dynamics upon the inclusion of different charged amphiphiles. Specifically, the results show that the inclusion of desipramine in lipid bilayers results primarily in a decrease in order of the PC lipid headgroup while leaving the conformation of the headgroup largely unchanged. In contrast, imipramine is shown to alter both conformation and order of the lipid headgroups. Changes in overall bilayer architecture were determined to be highly dependent on the TCA's effective charge and steric constraints. It is concluded that the ability of TCAs to induce lipidosis in vivo is not correlated in a straightforward manner with TCA-induced changes in overall bilayer architecture.

\section{EXPERIMENTAL}

\section{Materials}

The phospholipids, 1-Palmitoyl-2-oleoyl-sn-glycero-3-phosphatidylcholine (POPC) and 1-palmitoyl-2-oleoyl-sn-glycero-3-phosphatidylglycerol (POPG) were purchased from Avanti Polar Lipids (Alabaster, AL, USA) and used without further purification. The purity of the lipids was checked 
by thin-layer chromatography using $\mathrm{CH}_{3} \mathrm{Cl}-\mathrm{CH}_{3} \mathrm{OH}-\mathrm{H}_{2} \mathrm{O}$ $(85: 30: 3)$ as solvent system and iodine as visualization agent. Imipramine hydrochloride and desipramine hydrochloride were purchased from Sigma Chemical (St. Louis, MO, USA) and iminodibenzyl from Aldrich Chemical (Milwaukee, WI, USA). All chemicals were used without further purification.

\section{Sample preparation}

Oriented lipid bilayers were prepared by one of two methods, in which the only difference was the use of naphthalene, as described previously. ${ }^{33}$ Briefly, the drug compound was first dissolved in a chloroform solution containing the lipid of interest. The mixture was dried under a nitrogen flow and the residue was dissolved in 2:1 chloroform-methanol containing a 1:1 molar ratio of naphthalene, briefly sonicated and then spread on the surface of $2-6$ thin $(0.05 \times 11 \times 21 \mathrm{~mm})$ glass plates (Paul Marienfeld, Bad Mergentheim, Germany) that had previously been cleaned with chloroform. Plates were allowed to air-dry and residual traces of the organic solvents were removed at room temperature under high vacuum for at least $8 \mathrm{~h}$. Each plate contained $\sim 2 \mathrm{mg}$ of lipid. The removal of naphthalene was confirmed by ${ }^{1} \mathrm{H}$ solution NMR after dissolving the drug-lipid film in $\mathrm{CDCl}_{3}$. Samples were subsequently indirectly hydrated in a hydration chamber of $93 \%$ relative humidity using saturated $\mathrm{NH}_{4} \mathrm{H}_{2} \mathrm{PO}_{4}$ solution ${ }^{34}$ at $37^{\circ} \mathrm{C}$ for 2 days. After 2 days, a minimum of $28 \mathrm{~mol}$ of $4{ }^{\circ} \mathrm{C}$ water per mole of lipid was added to the sides of the plates so as to achieve complete hydration. ${ }^{35,36}$ The plates were stacked, wrapped with Parafilm, sealed in plastic bags (Plastic Bagmart, Marietta, GA, USA) and further equilibrated at $4{ }^{\circ} \mathrm{C}$ for several hours to 2 days. Complete hydration was monitored visually by the absence of a residual powder pattern.

Experiments were performed with the bilayer normal of the oriented sample parallel to the external magnetic field of the spectrometer. Multilamellar vesicle (MLV) samples were prepared by adding $100 \mathrm{wt} \%$ water $(\sim 42 \mathrm{~mol}$ of water per mole of lipid) to the dried lipid or lipid-drug mixture (5 mg for ${ }^{31} \mathrm{P}$ powder spectra and $20-60 \mathrm{mg}$ of lipid for ${ }^{14} \mathrm{~N}$ ), then homogenized using several liquid nitrogen freeze-room temperature thaw cycles. During the sample preparation, special care is needed with imipramine owing to its light sensitivity.

\section{NMR spectroscopy}

All experiments were performed on a Chemagnetics/Varian Infinity solid-state NMR spectrometer operating at resonance frequencies of $400.14,161.979$ and $28.9 \mathrm{MHz}$ for ${ }^{1} \mathrm{H},{ }^{31} \mathrm{P}$ and ${ }^{14} \mathrm{~N}$ nuclei, respectively. Experiments on oriented lipid bilayers were performed using laboratory-built double resonance $\left({ }^{1} \mathrm{H} /{ }^{14} \mathrm{~N}\right.$ and $\left.{ }^{1} \mathrm{H} /{ }^{31} \mathrm{P}\right)$ probes fitted with a five-turn rectangular coil made of a flat wire (2 mm wide) with a spacing of $1 \mathrm{~mm}$ between turns. Experiments on MLV samples were performed using a commercial Varian/Chemagnetics double resonance probe. All experiments were conducted at $35^{\circ} \mathrm{C}$ unless noted otherwise. ${ }^{31} \mathrm{P}$ spectra were acquired with a chemical shift echo pulse sequence $\left(90^{\circ}-\tau-180^{\circ}\right)$ under proton decoupling with a recycle delay of $3 \mathrm{~s}$. Typical values for the $90^{\circ}$ pulse width and $\tau$ delay were $3-4$ and $80 \mu$ s, respectively. A quadrupole echo pulse sequence $\left(90^{\circ}-\tau-90^{\circ}\right)$ was used to acquire the ${ }^{14} \mathrm{~N}$ magnetization under proton decoupling with a recycle delay of $3 \mathrm{~s}$. Typically, the values of the $90^{\circ}$ pulse width and $\tau$ were $3-5$ and $80 \mu$ s, respectively. ${ }^{1} \mathrm{H}$ decoupling r.f. field strengths of 35 and $65 \mathrm{kHz}$ were used during the signal acquisition in the ${ }^{31} \mathrm{P}$ and ${ }^{14} \mathrm{~N}$ experiments, respectively. Neither increasing the decoupling r.f. power nor implementing a TPPM decoupling sequence ${ }^{37}$ in the ${ }^{1} \mathrm{H}$ r.f. channel increased the resolution of the ${ }^{14} \mathrm{~N}$ spectral lines. For ${ }^{31} \mathrm{P}$ and ${ }^{14} \mathrm{~N}$ NMR experiments, $85 \%$ phosphoric acid and ammonium chloride solution were used as a standard reference samples ( 0 ppm). On average, 12000 transients were collected for ${ }^{14} \mathrm{~N}$ spectra whereas $<1000$ transients were required for the ${ }^{31} \mathrm{P}$ spectra. All of the spectra were obtained from a complex FID and data sets were zero-filled to 4096 points and processed with a maximum line broadening of $200 \mathrm{~Hz}$. The program IGOR Pro Version 3.1 for Windows (Wavemetrics, Lake Owesgo, OR, USA) was used to simulate powder spectra.

\section{RESULTS}

Solid-state NMR experiments were performed to obtain ${ }^{14} \mathrm{~N}$ and ${ }^{31} \mathrm{P}$ spectra of mixed POPC-POPG MLVs and oriented bilayers. This binary lipid system was selected for the following reasons: (1) POPC is a representative of lipids in the plasma membrane of eukaryotic cells and POPG is a suitable substitute for phosphatidylserine, the most abundant acidic lipid in mammalian cells, ${ }^{38}$ (2) in combination with POPG, POPC has been widely used in oriented bilayer studies of membrane-associated peptides ${ }^{16,39,40}$ and (3) both POPC and POPG are in the liquid crystalline phase at a physiological temperature [the temperature, $T_{\mathrm{C}}$, for the gel $\left(\mathrm{L}_{\beta}\right)$ to liquid crystalline $\left(\mathrm{L}_{\alpha}\right)$ phase transition is $-2.5 \pm 2.4^{\circ} \mathrm{C}$ for POPC and $0 \pm 1^{\circ} \mathrm{C}$ for POPG] and are assumed to be a good model for cellular membranes. ${ }^{41,42}$

Figure 3 shows ${ }^{31} \mathrm{P}$ spectra obtained at $35^{\circ} \mathrm{C}$ of MLVs and mechanically oriented bilayers of POPC, POPG and POPC-POPG mixtures with different molar ratios. Except for the spectrum of $4: 1$ POPC-POPG, all of the powder spectra can be fitted by a single powder pattern with the characteristic lineshape of the lipids in the liquid crystalline phase. Computer simulations were used to calculate CSA spans of $47.0 \pm 1.0 \mathrm{ppm}$ for pure POPC, $44.0 \pm 1.0 \mathrm{ppm}$ for the larger powder pattern in 4:1 POPC-POPG, $41.0 \pm 2.0 \mathrm{ppm}$ for $1: 1$ POPC-POPG, $38.5 \pm 1.0$ ppm for $1: 4$ POPC-POPG and $37.0 \pm 1.0 \mathrm{ppm}$ for pure POPG. The CSA spans for the pure lipids are in good agreement with previously reported values for phosphatidylcholines and phosphatidylglycerols. ${ }^{16,36,39}$ Given that the ${ }^{31} \mathrm{P}$ CSA spans of POPC and POPG powder spectra are markedly different (the span of the POPC spectrum is $10 \mathrm{ppm}$ larger than that of the POPG spectrum), a single CSA span observed in the ${ }^{31} \mathrm{P}$ spectra of the lipid mixtures indicates that the presence of POPC evokes a change in the headgroup conformation of POPG and vice versa. In contrast, the ${ }^{31} \mathrm{P}$ powder spectrum of $4: 1 \mathrm{POPC}-\mathrm{POPG}$ shows two distinct CSA spans with clear resolution on the 


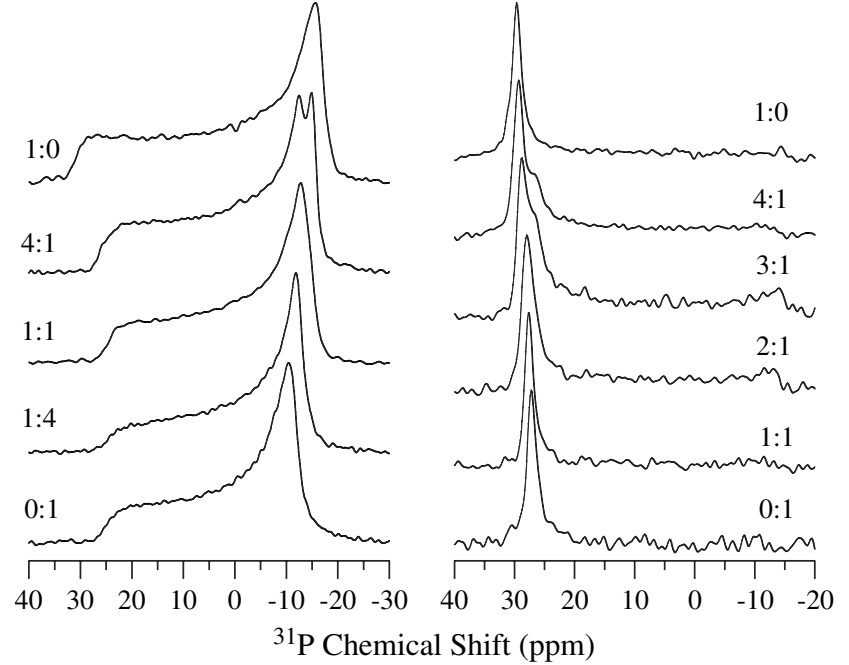

Figure 3. ${ }^{31} \mathrm{P}$ chemical shift spectra of POPC-POPG lipid $\operatorname{MLV}(A-E)$ and mechanically oriented $(F-K)$ samples. The POPC : POPG molar ratios are given on the traces.

perpendicular (higher field) edge of the powder spectrum. Considering the 4 : 1 POPC-POPG molar ratio used, the peak intensities of the two perpendicular edges are in discrepancy, with the edge at the lower field side being significantly more intense than expected if each CSA span was to be assigned exclusively to POPC and POPG. As shown in Fig. 3, this discrepancy was also observed in the oriented bilayer spectra where, for example, the area ratios of the two peaks in the $4: 1$ PC-PG and 3:1 PC-PG spectra were calculated to be $2.6: 1$ and $2.4: 1$, respectively. For the $1: 1$ POPC-POPG mixture, a narrow single line with chemical shift value close to that measured for the pure POPG spectrum is observed, confirming that (1) the POPC headgroup conformation is affected by the presence of neighboring negatively charged POPG molecules and (2) both lipids were homogenously mixed. In the spectrum of the 2: 1 POPC-POPG mixture, two lines appear to be merged in to one, as evidenced by the relatively broad line, suggesting that POPC and POPG are miscible but in the intermediate dynamics range. ${ }^{31} \mathrm{P}$ spectra of POPC-POPG lipid bilayers oriented with bilayer normal parallel to $B_{0}$ showed two peaks at the parallel edge (corresponding to two distinct lipid populations and two $\sigma_{/ /}$tensor components) for POPC: POPG lipid molar ratios $>2: 1$. The lipid populations giving rise to these lines are assigned to POPC- and POPGenriched domains and not to pure POPC and pure POPG domains since the ratio of the line intensities differs markedly from the stoichiometric ratio of lipids used in each sample (e.g. for 4:1 PC-PG the peak area ratio was 2.6:1). Hence it follows that the powder spectrum with a larger CSA span corresponds to a lipid population composed of POPC-enriched domains and the spectrum with a smaller CSA span corresponds to a lipid population of POPG-enriched domains.

To investigate the composition of each lipid domain and the relative affinities of each TCA for POPC and POPG, the ${ }^{31} \mathrm{P}$ powder spectra of POPC and POPG MLVs with $25 \mathrm{~mol} \%$ desipramine and imipramine incorporated were recorded (Fig. 4). Each spectrum exhibits a single powder pattern with lineshape characteristic of lipids in the $\mathrm{L}_{\alpha}$ fluid lamellar phase. The CSA spans were determined by computer simulation to be $49.0 \pm 1.0,57.0 \pm 1.0,38.0 \pm 1.0$ and $44.0 \pm 1.0 \mathrm{ppm}$ for POPC-desipramine [Fig. 4(A)], POPC-imipramine [Fig. 4(B)], POPG-desipramine [Fig. 4(C)] and POPG-imipramine [Fig. 4(D)], respectively. By comparing the CSA spans of the ${ }^{31} \mathrm{P}$ spectra of POPC and POPG in the absence of any drugs (Fig. 3) with those in the presence of drugs (Fig. 4), it is possible to conclude that the incorporation of either desipramine or imipramine changes the lipid headgroup conformation/dynamics resulting in an increase in the CSA span of POPC and POPG (see Table 1). Importantly, the increases in the CSA spans for both lipids are much more dramatic upon incorporation of imipramine than desipramine as, for example, the CSA span of POPC MLVs increases from $47.0 \pm 1.0 \mathrm{ppm}$ without drug to $49.0 \pm 1.0 \mathrm{ppm}$ with desipramine and to $57.0 \pm 1.0 \mathrm{ppm}$ with imipramine. No change was observed in the spectrum of POPC and POPG MLVs with iminodibenzyl incorporated (spectra not shown).

Figure 5 shows the powder and oriented ${ }^{31} \mathrm{P}$ chemical shift spectra of 4:1 POPC-POPG mixtures with drugs incorporated. As was previously seen in the spectrum of 4:1 POPC-POPG MLVs, all of the powder spectra of 4:1 POPC-POPG MLVs with 25\% molar drugs are composed of two powder patterns with two resolvable perpendicular edges. As expected, the spectra of the oriented bilayers of analogous lipid and drug composition also show two well-resolved parallel edges. The CSA spans for the individual powder patterns in the ${ }^{31} \mathrm{P}$ powder spectrum of $4: 1 \mathrm{POPC}-\mathrm{POPG}$ with $25 \mathrm{~mol} \%$ desipramine incorporated [Fig. 5(A)] were calculated to be

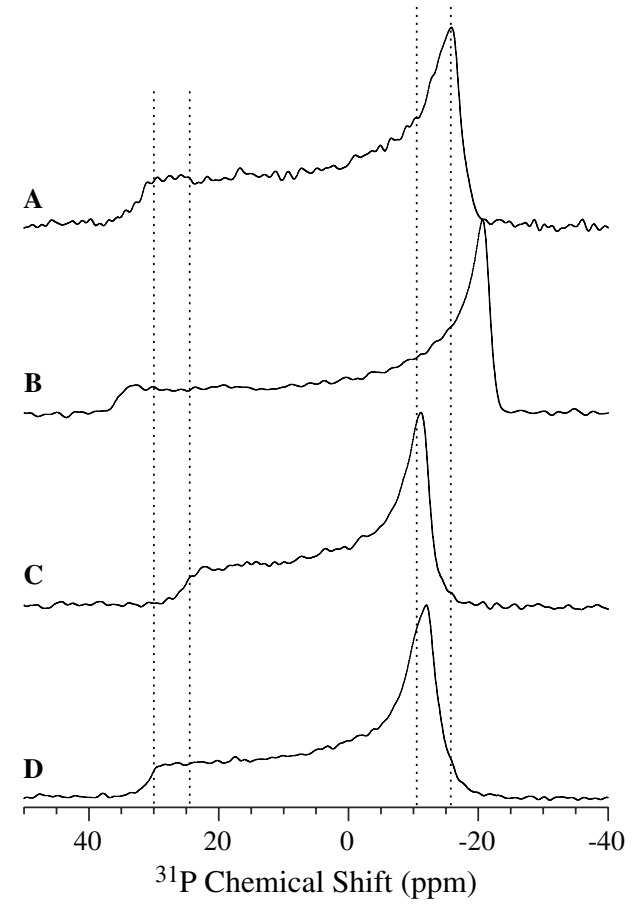

Figure 4. ${ }^{31} \mathrm{P}$ chemical shift powder spectra of POPC or POPG with 25 mol\% drug incorporated. (A) POPC-desipramine;

(B) POPC-imipramine; (C) POPG-desipramine;

(D) POPG-imipramine. Dashed lines are to guide the eye. 
Table 1. ${ }^{14} \mathrm{~N}$ quadrupole splittings and ${ }^{31} \mathrm{P}$ chemical shift anisotropy spans for MLV samples with and without TCA drugs

\begin{tabular}{|c|c|c|c|c|}
\hline Inclusion & Lipids & $\begin{array}{l}{ }^{31} \mathrm{P} \Delta \sigma \\
(\mathrm{ppm})\end{array}$ & $\begin{array}{c}{ }^{14} \mathrm{~N} \Delta v_{\mathrm{Q}} \\
(\mathrm{kHz})^{\mathrm{b}}\end{array}$ & Fig. \\
\hline \multirow[t]{5}{*}{ None } & PC & $47.0 \pm 1.0$ & $10.8 \pm 0.2$ & 3 \\
\hline & PC-PG $(4: 1)$ & $44.0 \pm 1.0^{\mathrm{a}}$ & $11.5 \pm 0.2$ & $3,6(\mathrm{~A})$ \\
\hline & PC-PG $(1: 1)$ & $41.0 \pm 1.0$ & n.r. & 3 \\
\hline & PC-PG $(1: 4)$ & $38.5 \pm 1.0$ & n.r. & 3 \\
\hline & PG & $37.0 \pm 1.0$ & n.a. & 3 \\
\hline \multirow[t]{4}{*}{$25 \%$ Desipramine } & PC-PG $(4: 1)$ & $42.0 \pm 1.0$ & $7.5 \pm 0.2$ & $5(\mathrm{~A}), 6(\mathrm{~B})$ \\
\hline & & $39.0 \pm 1.0$ & & \\
\hline & PC & $49.0 \pm 1.0$ & n.r. & $4(\mathrm{~A})$ \\
\hline & PG & $38.0 \pm 1.0$ & n.a. & $4(\mathrm{C})$ \\
\hline \multirow[t]{4}{*}{$25 \%$ Imipramine } & PC-PG $(4: 1)$ & $57.0 \pm 1.0$ & $8.5 \pm 0.2$ & $5(B), 6(C)$ \\
\hline & & $43.7 \pm 1.0$ & & \\
\hline & PC & $57.0 \pm 1.0$ & n.r. & $4(\mathrm{~B})$ \\
\hline & PG & $44.0 \pm 1.0$ & n.a. & $4(\mathrm{D})$ \\
\hline $25 \%$ Iminodibenzyl & PC-PG $(4: 1)$ & $48.0 \pm 2.0$ & $11.4 \pm 0.2$ & $5(C), 6(D)$ \\
\hline
\end{tabular}

${ }^{a}$ Only the largest CSA span could be measured.

${ }^{b}$ n.r., Not recorded; n.a., not applicable.

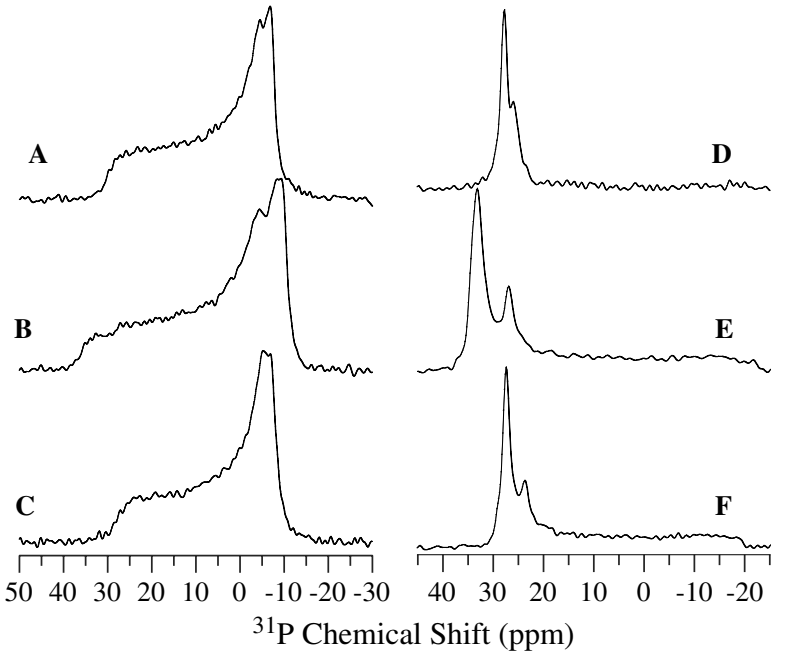

Figure 5. ${ }^{31} \mathrm{P}$ chemical shift spectra of $4: 1 \mathrm{POPC}-\mathrm{POPG}$ MLV (left) and mechanically oriented (right) samples at various molar concentrations of a drug: (A) and (D) $25 \mathrm{~mol} \%$ desipramine; (B) and (E) 25 mol\% imipramine; (C) and (F) 25 mol\% iminodibenzyl.

$42.0 \pm 1.0$ and $39.0 \pm 1.0 \mathrm{ppm}$. In comparison, the powder spectrum of the same lipid mixtures with $25 \mathrm{~mol} \%$ imipramine [Fig. 5(B)] was deconvoluted into two powders with increased CSA spans relative to those calculated for desipramine, specifically $57.0 \pm 1.0$ and $43.7 \pm 1.0 \mathrm{ppm}$. Furthermore, the CSA span of the powder spectra of POPC MLVs with desipramine incorporated is significantly larger than the corresponding larger CSA span observed in the spectra of the binary lipid mixture with desipramine incorporated $(42.0 \pm 1.0$ versus $49.0 \pm 1.0 \mathrm{ppm})$. In contrast, the spans of the POPC powder spectra in both the binary and ternary lipid-imipramine mixtures are equal within error $(57 \pm 1.0 \mathrm{ppm})$.
At the very least, these results indicated that desipramine and imipramine interact with the lipids in a distinguishable manner and that in the desipramine case the effect of the drug on the overall bilayer architecture results from a combination of three pairwise interactions (PC-drug, PG-drug and PC-PG) that are not independent. A more detailed analysis of this observation is given in the Discussion section. The incorporation of iminodibenzyl results in only a slight broadening of the spectral lines without changing the ${ }^{31} \mathrm{P}$ CSA span $(48.0 \pm 2.0 \mathrm{ppm})$. In summary, all of the spectra confirmed the above-outlined trends for pure lipid systems. Briefly, (1) all of the spectra of 4:1 POPC-POPG mixture exhibit two peaks at the parallel edge in oriented spectra and two peaks at the perpendicular edge in powder spectra; (2) changes in the value of $\sigma_{/ /}$in the powder spectrum (the edge at lower field) and the chemical shift values of the peak at the lower field in oriented spectra for bilayers without drug and with $25 \mathrm{~mol} \%$ desipramine or iminodibenzyl are small; (3) the $\sigma_{/ /}$tensor components for both lipid bilayer populations with $25 \mathrm{~mol} \%$ imipramine incorporated are significantly larger and the corresponding peaks are broader than those observed from all other spectra; (4) all of the spectra of oriented bilayers with and without drug exhibit peak intensity ratios markedly different from the stochiometric ratio of 4:1 PC:PG. Specifically, the peak area ratios for 4:1 POPC-POPG bilayers with $25 \mathrm{~mol} \%$ desipramine [Fig. 5(D)], imipramine [Fig. 5(E)] and iminodibenzyl [Fig. 5(F)] were calculated to be 1.9:1, 1.8:1 and $2.8: 1$, respectively, supporting the contention that the lipid populations giving rise to each peak are not due to separate POPC and POPG populations but to the existence of two distinct lipid domains.

Together, the results presented thus far of ${ }^{31} \mathrm{P}$ chemical shift spectra of mixed and individual lipids with drugs allow us to draw a number of important conclusions. First, it is verified that both desipramine and imipramine 
are most likely positively charged at neutral $\mathrm{pH}$, as expected based on the reported $\mathrm{p} K_{\mathrm{a}}$ values of 9.5 and 10.2 for these compounds. ${ }^{43}$ This conclusion is drawn from previous studies where an increase in the ${ }^{31} \mathrm{P}$ CSA span for phosphatidylcholines was interpreted in terms of a reorientation of the $\mathrm{P}-\mathrm{N}$ dipole vector towards the water layer caused by a repulsion between a cationic amphiphile and the lipid choline site. ${ }^{32}$ Second, $25 \mathrm{~mol} \%$ desipramine and imipramine are fully miscible in both lipids as a single CSA pattern describes the powder spectra. Third, by comparison with the ${ }^{31} \mathrm{P}$ powder spectra of $25 \mathrm{~mol} \%$ desipramine and imipramine in 4:1 POPC-POPG [Fig. 5(A) and (B), respectively], it is possible to assign the outer powder pattern (the one with larger CSA span) to a lipid domain enriched in POPC and the inner powder pattern to a lipid domain enriched in POPG in both cases. Finally, a comparison of CSA spans of ${ }^{31} \mathrm{P}$ powder spectra of POPC, 4:1 POPC-POPG and POPC with $25 \mathrm{~mol} \%$ desipramine incorporated shows that desipramine and POPG change the ${ }^{31} \mathrm{P}$ CSA span of POPC in opposite directions. Notably, the increase in the ${ }^{31} \mathrm{P}$ CSA span of POPC is even more pronounced upon introduction of imipramine.

To investigate further the effect of POPG and drugs on the POPC conformation and dynamics in a more specific manner, the ${ }^{14} \mathrm{~N}$ powder spectra of $4: 1$ POPC-POPG without drug and with $25 \mathrm{~mol} \%$ desipramine, imipramine and iminodibenzyl incorporated were recorded [Fig. 6(A)-(D)]. The ${ }^{14} \mathrm{~N}$ powder spectra POPC (spectrum not shown) and 4:1 POPC-POPG [Fig. 6(A)] MLVs in the $\mathrm{L}_{\alpha}$ phase showed significantly narrower quadrupolar splittings than those predicted for an immobile POPC (a quadrupole splitting

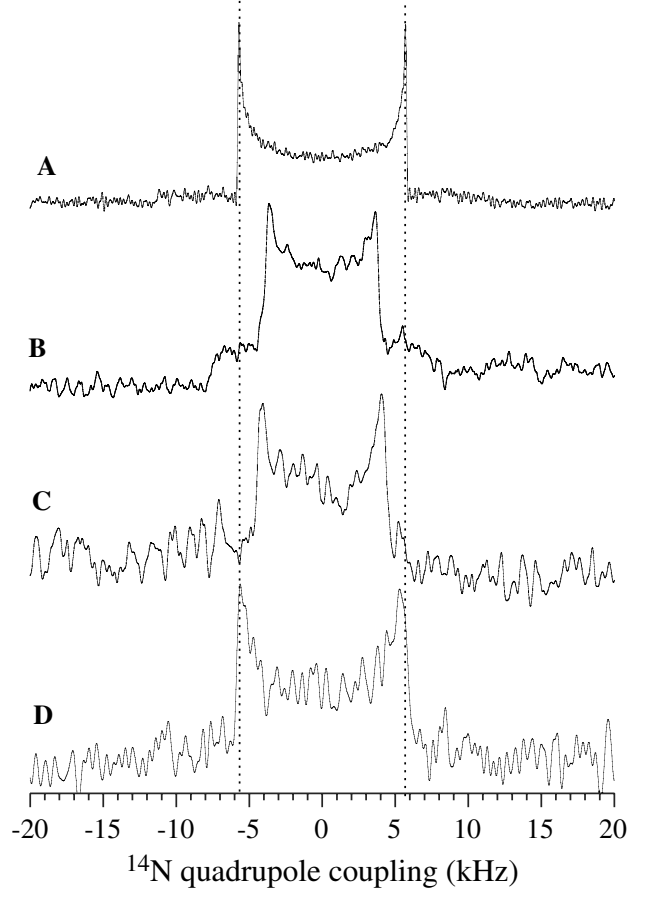

Figure 6. ${ }^{14} \mathrm{~N}$ quadrupole coupling powder spectra of $4: 1$ POPC-POPG at various molar concentrations of a drug: (A) without drug; (B) 25 mol\% desipramine; (C) 25 mol\% imipramine; (D) 25 mol\% iminodibenzyl. Dashed lines are to guide the eye. of $101.25 \mathrm{kHz}$ ). The separations between the peak maxima $\left(\Delta v_{\mathrm{Q}}\right)$ of POPC (spectrum not shown) and $4: 1$ POPC-POPG [Fig. 6(A)] are $10.8 \pm 0.2$ and $11.5 \pm 0.2 \mathrm{kHz}$, respectively, in agreement with previous studies. ${ }^{24,31}$ This averaging of the quadrupole interaction is achieved by the axial rotation of the lipid molecules at a rate faster than the effective static quadrupole coupling constant for the ${ }^{14} \mathrm{~N}$ nucleus in the choline headgroup. The addition of desipramine [Fig. 6(B)] and imipramine [Fig. 6(C)] to 4:1 POPC-POPG at a $25 \mathrm{~mol} \%$ concentration resulted in noticeable changes in the quadrupole splitting $\left(\Delta v_{\mathrm{Q}}=7.5 \pm 0.2\right.$ and $8.5 \pm$ $0.2 \mathrm{kHz}$, respectively). In contrast, incorporation of the same concentration of iminodibenzyl in 4:1 POPC-POPG MLVs [Fig. 6(D)] revealed no change in the quadrupole splitting of the choline group. A decrease in the quadrupole splitting is interpreted as a decrease in the order parameter for the nitrogen nucleus at the POPC choline site. As such, we conclude that the inclusion of desipramine and imipramine at $25 \mathrm{~mol} \%$ into $4: 1$ POPC-POPG mixture decreases the order parameter of the choline headgroup, whereas the addition of iminodibenzyl at the same molar concentration broadens the lines without changing, to a first approximation, the order parameter. Incorporation of desipramine results in a larger decrease in the order parameter at the choline headgroup than imipramine. Finally, given that approximately the same weight of lipid was present in all samples and the number of scans was approximately the same for all ${ }^{14} \mathrm{~N}$ spectra obtained, we interpret the visible increase in the line broadening upon the inclusion of drugs as a noticeable change in the ${ }^{14} \mathrm{~N}$ relaxation time.

To test if the effect of desipramine on the ${ }^{14} \mathrm{~N}$ order parameter of the choline group of POPC was concentration dependent, the ${ }^{14} \mathrm{~N}$ spectra of mechanically oriented 4:1 POPC-POPG bilayers in the presence of different desipramine concentrations were recorded (Fig. 7). The quadrupole splittings ( $\Delta v_{\text {oriented }}$ ) observed from the oriented spectra were $23.0 \pm 0.2,16.2 \pm 0.2,13.6 \pm 0.4,11.6 \pm 0.4$ and $10.2 \pm 0.6 \mathrm{kHz}$ for $4: 1$ POPC-POPG with desipramine concentrations of 3,20,30, 40 and $50 \mathrm{~mol} \%$, respectively.

The ratio of $\Delta v_{\text {oriented }}$ to $\Delta v_{\mathrm{Q}}$ is related to an angle, $\alpha$, defined by the following expression:

$$
\frac{\Delta v_{\text {oriented }}}{\Delta v_{\mathrm{Q}}}=\left|\overline{3 \cos ^{2} \alpha}-1\right|
$$

where $\alpha$ is the angle between the primary quadrupole interaction averaging axis and the bilayer normal (parallel to $B_{0}$ ). Because this averaging axis has been shown previously to be approximately collinear with the lipid symmetry axis, it is possible to estimate the orientation of POPC with respect to the bilayer normal at different desipramine concentrations. Since $\Delta v_{\text {oriented }}$ for 4:1 POPC-POPG bilayers with $25 \mathrm{~mol} \%$ desipramine incorporated was not directly measured, this value is calculated to be $14.9 \pm 0.3 \mathrm{kHz}$ by averaging the $\Delta v_{\text {oriented }}$ values obtained from 20 and $30 \mathrm{~mol} \%$ desipramine incorporated. Substitution of this value and of the $\Delta v_{\mathrm{Q}}$ value observed in the ${ }^{14} \mathrm{~N}$ powder spectra of $4: 1$ POPC-POPG with $25 \mathrm{~mol} \%$ desipramine $(7.5 \mathrm{kHz})$ into Eqn (1) yields $\alpha=0$, indicating that the incorporation of $25 \mathrm{~mol} \%$ desipramine into $4: 1$ POPC-POPG bilayers leaves 


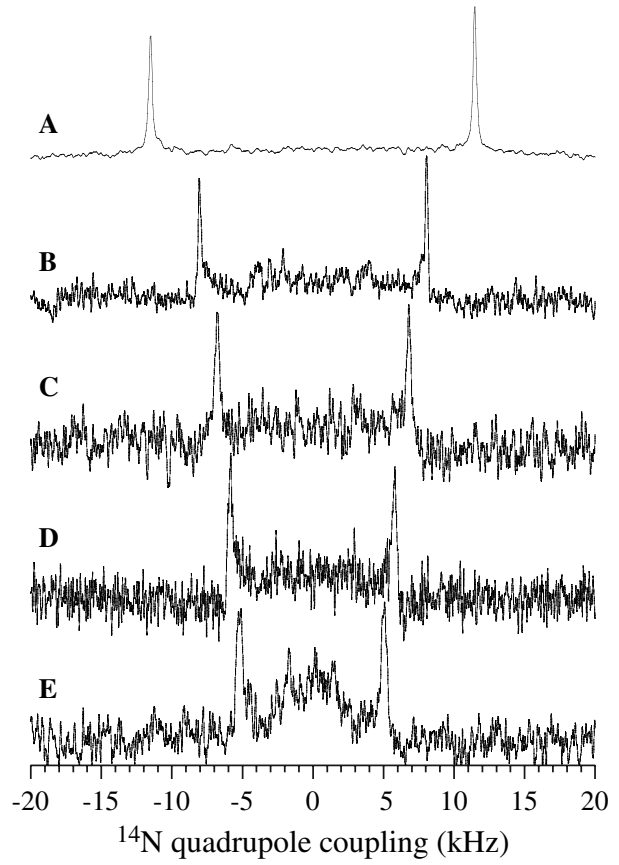

Figure 7. ${ }^{14} \mathrm{~N}$ quadrupole coupling spectra of mechanically oriented $4: 1$ POPC-POPG at various molar concentrations of desipramine: (A) 3; (B) 20; (C) 30; (D) 40; (E) 50 mol\%.

POPC with its symmetry axis oriented parallel to the bilayer normal. This leads us to conclude that desipramine imparts a disorder on the POPC headgroup while leaving the lipid orientation unchanged. This conclusion is consistent with the data collected by Rothgeb and Oldfield on oriented bilayers of 1:11,2-dimyristoyl-sn-glycero-3-phosphatidylcholine (DMPC)-desipramine. ${ }^{1}$ The appearance of signal intensity at approximately half of the value of $\Delta v_{\text {oriented }}$ in bilayers containing $40 \mathrm{~mol} \%$ (from -3 to $+3 \mathrm{kHz}$ ) and $50 \mathrm{~mol} \%$ (from -2.5 to $+2.5 \mathrm{kHz}$ desipramine [see Fig. 7(D) and (E), respectively]) indicates the presence of small amounts of unoriented bilayers. It is also noticeable that at these desipramine concentrations (40 and $50 \mathrm{~mol} \%$ ) the ${ }^{14} \mathrm{~N}$ lines are broader, in agreement with the observed broadening of the ${ }^{31} \mathrm{P}$ spectra of $4: 1$ POPC-POPG bilayers with $50 \mathrm{~mol} \%$ desipramine incorporated (spectra not shown).

To distinguish a decrease in the ${ }^{14} \mathrm{~N}$ order parameter due to an increase in disorder from a change in the POPC headgroup conformation, the ${ }^{31} \mathrm{P}$ chemical shift spectra of oriented 4:1 POPC-POPG bilayers with bilayer normal parallel to $B_{0}$ were recorded in the presence of desipramine concentrations at which desipramine was completely soluble (below $30 \mathrm{~mol} \%$, Fig. 8). As the concentration of desipramine is increased from 0 to $25 \mathrm{~mol} \%$, the chemical shift and width of the peak at lower field, corresponding to the parallel edge of POPC-enriched domain, remain relatively unchanged. We conclude that the conformation of the POPC headgroup in the phosphate region in the presence of miscible concentrations of desipramine remains approximately the same and that the observed decrease in the ${ }^{14} \mathrm{~N}$ quadrupole splitting of POPC in the presence of desipramine can be assigned primarily to a disordering effect. Interestingly, the chemical shift value and its intensity of the peak at higher field in Fig. 8 vary more drastically with desipramine concentration. Importantly, the

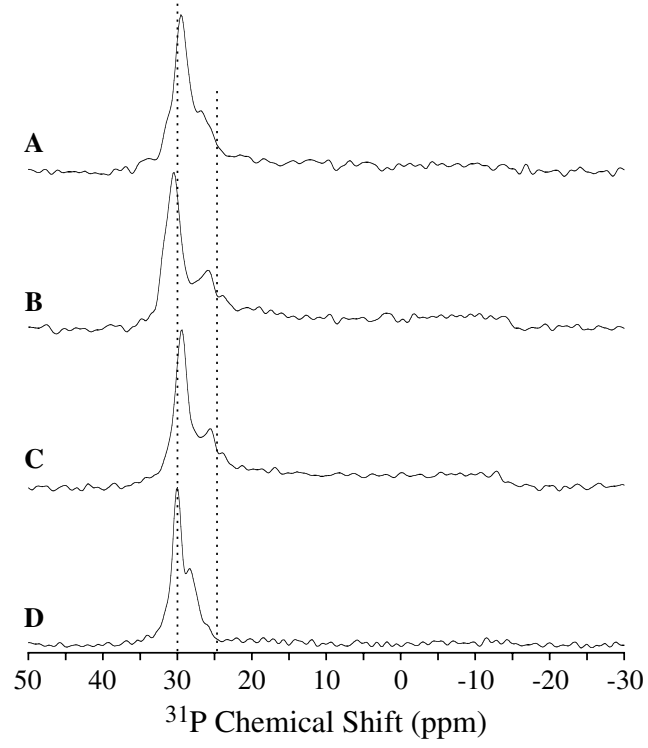

Figure 8. ${ }^{31} \mathrm{P}$ chemical shift spectra of mechanically oriented 4:1 POPC-POPG at various molar concentrations of desipramine: (A) 3; (B) 10; (C) 20; (D) 25 mol\%. Dashed lines are to guide the eye.

ratio of the intensities of the peaks never approaches $4: 1$, the POPC:POPG stoichiometric ratio used in the sample, indicating that the chemical shift and intensity of the peak at higher field are a measure of the ternary interaction among drug, POPC and POPG. In analogy with the observed effect of increasing the concentration of POPG in POPC-POPG MLV samples (Fig. 3), we propose that the absolute difference in chemical shift values of the two peaks is indicative of the miscibility of POPG in POPC bilayers and therefore of each lipid domain composition. The apparent lack of a trend in this absolute difference of chemical shifts with increasing desipramine concentration is interpreted as a signal that the composition of the two lipid domains is constantly changing without reaching a steady state in the time of the experiment. This issue will be addressed in further detail in the Discussion section. A summary of the results obtained for MLV samples is shown in Table 1.

\section{DISCUSSION}

It has been demonstrated previously that the TCA class of compounds interacts with the lipid bilayer. ${ }^{1-4}$ The ${ }^{31} \mathrm{P}$ chemical shift spectra of binary lipid systems (charged drug with POPC or POPG) in the present study clearly support this conclusion by demonstrating that both imipramine and desipramine interact with POPC in addition to POPG as reflected by the changes in the CSA span. However, this information does not address the question of how the interaction of different TCAs with the lipid membrane may contribute to the onset of distinguishing physiologically observed direct and secondary effects by altering the membrane lipid dynamics or conformation. This is precisely the goal of this study. To date, the study of lipid-TCA interactions had been reduced to observations on binary systems (TCA + PC) with a single probe (either ${ }^{14} \mathrm{~N}$ or $\left.{ }^{31} \mathrm{P}\right) .{ }^{1,2}$ 
To address the question posed, we made a comparison of the effect of TCAs on ternary systems (TCA + two lipids) with two different probes. This approach allowed us to determine that the two TCAs, imipramine and desipramine, alter the membrane structure and dynamics in distinct ways that may be correlated with the observed differences in TCA-related secondary effects. Specifically, it is shown that the effect of the TCA on the lipid bilayer is highly dependent on the TCA's effective charge and also steric constraints.

Iminodibenzyl, a structural homologue of imipramine and desipramine, retains the tricyclic structure of all TCA compounds, but it is missing a charged acyl chain. Incorporation of this compound into 4:1 POPC-POPG system did not change the ${ }^{14} \mathrm{~N}$ order parameter of the choline group of POPC [Fig. 6(D)]. In addition, the ${ }^{31} \mathrm{P}$ CSA span calculated from the powder spectrum of $4: 1$ POPC-POPG with $25 \mathrm{~mol} \%$ iminodibenzyl incorporated [Fig. 5(C)] was shown to be equal, within experimental error, to that observed for the same lipid composition without the drug. In support of an unchanged lipid bilayer conformation and dynamics upon addition of iminodibenzyl, the ${ }^{31} \mathrm{P}$ spectra of oriented 4:1 POPC-POPG bilayers with and without iminodibenzyl showed little change in the chemical shift value of both peaks. Iminodibenzyl is thus shown to act as an inert spacer between lipid molecules. This observation is consistent with the absence of a reported role of iminodibenzyl in induction of lipidosis.

In contrast to iminodibenzyl, both desipramine and imipramine have charged acyl chains and induce lipidosis in vivo (more acute for desipramine than imipramine).$^{7-11}$ In a previous study using ${ }^{13} \mathrm{C}$ and ${ }^{2} \mathrm{H} \mathrm{NMR},{ }^{44}$ desipramine and imipramine were shown to partition into the lipid bilayer inducing the largest disordering effect on the lower acyl chain carbons (6-14 in DMPC), which indicates that both drugs penetrate the bilayer only down to the sixth carbon on the lipid acyl chain. This disordering effect was measured to be larger for desipramine than imipramine. It has also been proposed that locating desipramine or imipramine at the depth of carbon- 6 would position the charged amine at a short distance from either the lipid $\mathrm{O}_{22(32)}$ or $\mathrm{O}_{13}$ (Fig. 2) and a hydrogen bond forms between the charged drug acyl chain and these lipid oxygens, ${ }^{45,46}$ also in agreement with ${ }^{13} \mathrm{C}$ and ${ }^{1} \mathrm{H}$ NMR results from studies on detergent micelles and liposomes that position the charged acyl chain of TCAs at the air-water interface. ${ }^{47,48}$ Therefore, it is not surprising that both imipramine and desipramine interact with the lipid headgroups and that the extent of the drug-induced bilayer disordering effect is correlated with the effective charge of the drug. The results presented in this study on the effect of the incorporation of these two compounds into 4:1 POPC-POPG bilayers support both of these conclusions. More importantly, our results show that both imipramine and desipramine alter the bilayer conformation and dynamics in distinct ways.

Incorporation of desipramine into 4:1 POPC-POPG mixtures is observed to have a large disordering effect on the choline headgroup of POPC while leaving the conformation of the POPC and POPG headgroup at the phosphate site unchanged. In support of this conclusion, the ${ }^{14} \mathrm{~N}$ quadrupole splitting of the POPC choline headgroup in POPC-POPG mixtures decreases from $11.5 \pm 0.2$ to $7.5 \pm 0.2 \mathrm{kHz}$ on addition of $25 \mathrm{~mol} \%$ desipramine while leaving the lipid symmetry axis parallel to the bilayer normal. The decrease in the quadrupole splitting was observed to be almost linearly dependent on the desipramine concentration. The decrease in the quadrupole splitting and order parameter of the ${ }^{14} \mathrm{~N}$ nucleus could not be assigned to a change in headgroup conformation since (1) the calculated ${ }^{31} \mathrm{P}$ CSA spans of POPC-POPG mixtures without drug and with 25 and $50 \mathrm{~mol} \%$ desipramine incorporated are equal within experimental error and (2) the ${ }^{31} \mathrm{P}$ chemical shifts of the peaks at higher field in oriented 4:1 POPC-POPG bilayers with desipramine concentrations ranging from 0 to $25 \mathrm{~mol} \%$ are equal within experimental error. Importantly, it was the use of ${ }^{14} \mathrm{~N}$ as a probe of the TCA-lipid interaction that allowed the determination of a strong effect of desipramine on the bilayer architecture as the ${ }^{31} \mathrm{P}$ spectra show only small changes.

In comparison with desipramine, incorporation of imipramine into 4:1 POPC-POPG mixtures is observed to decrease the ${ }^{14} \mathrm{~N}$ quadrupole splitting (or the order parameter) of the POPC choline headgroup to a smaller extent than desipramine while concurrently increasing the ${ }^{31} \mathrm{P}$ CSA span. Because both the ${ }^{31} \mathrm{P}$ CSA span and the ${ }^{14} \mathrm{~N}$ order parameter change, it is not possible to assign the decrease in the ${ }^{14} \mathrm{~N}$ order parameter solely to an increase in the choline headgroup disorder. A change in conformation of the lipid headgroup could also result in a decrease of the POPC choline quadrupole splitting. The increase in CSA span upon inclusion of imipramine occurs concurrently with homogenous line broadening, which is indicative of a change in headgroup dynamics as seen at the phosphate site. In conclusion and in comparison with desipramine, the inclusion of imipramine in $4: 1$ POPC-POPG bilayers is observed to have an effect on the conformation and/or dynamics at both the choline and phosphate sites whereas desipramine is observed to increase mostly the disorder of the choline headgroup while leaving the conformation of the phosphate site largely unchanged.

The conclusion presented above was made based on the effect of each drug on POPC bilayers containing a smaller concentration of POPG. An assessment of the effect of each drug on the POPG headgroup is difficult to make since in mixed POPC-POPG the ${ }^{31} \mathrm{P}$ resonances of POPG have a contribution from POPC. The best evidence that this overlap exists is that none of the ${ }^{31} \mathrm{P}$ spectra of oriented 4:1 POPC-POPG showed the ratio of the intensities of the two peaks approaching to $4: 1$ ratio used in the mixture. Instead, the ratios were always in the range of $2.2: 1-2.8: 1$. Since the ${ }^{31} \mathrm{P}$ CSA span of POPG remains unchanged within experimental error with and without desipramine, to a first approximation, the effect of POPC and desipramine on the POPG headgroup is the same. In comparison, imipramine clearly changes the POPG headgroup conformation and dynamics as shown by a significant increase in the ${ }^{31} \mathrm{P}$ CSA span of POPG with and without imipramine. In 4:1 POPC-POPG bilayers with drug, the chemical shift difference between the two peaks is taken as a measure of the miscibility of POPC in POPG. An increase in the 
difference relative to the spectra of drug-free bilayers as seen, for example, in bilayers with $25 \mathrm{~mol} \%$ imipramine suggests that imipramine makes POPC less miscible in POPG most likely because the positively charged imipramine competes with POPC for an interaction with POPG. Noticeably, the miscibility of POPC in POPG is also, but to a much lesser extent, dependent on the desipramine concentration.

Guided by the results from previous studies that showed that both desipramine and imipramine insert into the lipid bilayer positioning the charged acyl chain at the depth of $\mathrm{O}_{13}$ or $\mathrm{O}_{22(32)}$ (Fig. 2) and possibly making a hydrogen bond with these sites, the new results presented here allow us to formulate a crude structural model for the interaction of TCA with the lipid headgroup. Computer simulation studies of pure lipid bilayers of phosphatidylcholines indicated that the primary interactions between lipids headgroups are hydrogen bonds and charge associations between the choline nitrogen and $\mathrm{O}_{13}$ and $\mathrm{O}_{22(32)} \cdot{ }^{19}$ Our data suggest that the acyl chains in both imipramine and desipramine whose charged amine resembles the choline headgroup of POPC interact with the phosphate site of both POPC and POPG. In both cases, we propose that the interaction of the charged amine of imipramine and desipramine with the phosphate sites of POPG and POPC makes the phosphate sites of these lipids less available for lipid 'cross-linking'. Visually this can be seen as a 'release' of the choline headgroup or $\mathrm{P}-\mathrm{N}$ vector (Fig. 2), which allows it to have a greater amplitude of motion reported by the ${ }^{14} \mathrm{~N}$ spectra as an increase in disorder (decrease of order parameter). Electrostatically, the repulsion between the charged drug amine and the choline headgroup can also contribute to this effect. The more unshielded charge of desipramine is able to substitute, to a very good approximation, the interaction of POPC choline with POPG and/or POPC phosphate. This conclusion is supported by equal ${ }^{31} \mathrm{P} C S A$ spans within experimental errors for spectra of POPG in the presence of POPC or desipramine and by the relatively unchanged chemical shift of POPC in oriented bilayers of $4: 1$ POPC-POPG with and without desipramine. Imipramine is different from desipramine in that its more sterically shielded charge does not substitute well for the interaction of the choline POPC with the phosphate groups of either POPC or POPG. The dramatic increase in the ${ }^{31}$ PCSA span of $4: 1$ POPC-POPG mixture and in the chemical shifts of both peaks in oriented lipid bilayers upon the inclusion of imipramine supports this conclusion. In summary, this model confirms that there is a correlation between the effective charge of TCA as well as steric constraints and observed changes in bilayer architecture. Furthermore, this model argues for a need to probe both regions (phosphate and choline) of the lipid headgroup to ascertain the effect of amphiphiles on bilayer architecture.

It should be mentioned that the drug concentrations used in this study are orders of magnitude above what is seen at the macroscopic level in vivo. ${ }^{7-11}$ However, it is possible that lower microscopic drug concentrations may induce significant changes in bilayer architecture such as those outlined in this study. Furthermore, it is also conceivable that if secondary effects (e.g. lipidosis) resulting from the ingestion of TCA-like compounds are induced by the inability of the phospholipase to interact with the membrane, then the membrane changes necessary to abolish those interactions need only be much smaller than those observed spectroscopically in this study. For these reasons, this study provides a step forward towards understanding the interactions of TCAs with lipid bilayers that may be related to the ability of the compounds to induce lipidosis. Finally, we conclude that, unless the propensity of TCAs to induce lipidosis is shown in subsequent studies to be well correlated with the ability of these compounds to increase the disorder of the lipid bilayers closest to the cytoplasm (e.g. choline sites in phosphatidylcholines) while leaving the conformation of the phosphate region of the lipid headgroup largely unchanged, the effects of TCA addition to lipid bilayers does not explain lipidosis as we observe that imipramine imparts more dramatic changes in the overall bilayer architecture than desipramine.

\section{CONCLUSIONS}

The combined application of ${ }^{14} \mathrm{~N}$ and ${ }^{31} \mathrm{P}$ solid-state NMR experiments to study the interaction of antidepressants with lipids was shown to provide a clearer picture of the drug-induced changes in the lipid bilayer. ${ }^{49}$ In this model, inclusion of charged TCA was shown to change lipid conformation and/or dynamics. Specifically, addition of the charged TCA desipramine to $4: 1$ POPC-POPG mixtures was shown to increase the choline site disorder, leaving the conformation of the phosphate region of POPC and of the POPG headgroups largely unchanged. The incorporation of imipramine into lipid bilayers was shown to modulate both lipid conformation and dynamics. The changes in bilayer architecture imposed by the addition of desipramine and imipramine could modify the interactions between the bilayer and other membrane-associated proteins, possibly contributing to such TCA side-effects as lipidosis. However, it is noted that imipramine, which has a lower propensity to induce lipidosis in vivo, has a larger effect on overall bilayer architecture than desipramine, thereby arguing that TCA-mediated changes in lipid bilayer conformation and dynamics and the ability to induce lipidosis are not straightforward. In general, this study provides a generalized structural picture for the ability of amphiphiles with charged 'headgroups' capable of inserting into the lipid bilayer to change the properties of the membrane. Further studies on how the incorporation of TCA mediates the binding constants and activities of proteins that require interactions with anionic lipids (e.g. DnaA, antimicrobial peptides and phospholipases) will be particularly helpful in confirming the results outlined in this paper.

The present study has also demonstrated that ${ }^{14} \mathrm{~N}$ NMR has the potential of being a very useful probe to study model membranes. Its natural abundance (99.63\%) in a wide variety of phospholipids, particularly among the nitrogenous-base containing glycerophosphatides such as PC, makes it particularly attractive. Since the biological function of peptides, proteins or drugs in the cell membrane is highly dependent on the lipid environment, it will be useful to study the peptide or protein interactions with 
the lipid bilayers under various experimental conditions. Most importantly, the use of ${ }^{14} \mathrm{~N}$ NMR for probing lipid bilayers provides previously unavailable information. As an example, this TCA-lipid interaction study indicates that ${ }^{14} \mathrm{~N}$ solid-state NMR is capable of differentiating between the effects on bilayer architecture of compounds whose charge is only slightly different, as is the case for desipramine and imipramine. We trust that the advent of higher magnetic fields, the development of new solid-state NMR techniques and significant improvements in preparing membranemimicking model samples will lead to more successful studies of ${ }^{14} \mathrm{~N}$ NMR spectroscopy applied to non-isotropic biological samples.

\section{Acknowledgments}

This research was supported in part by a National Science Foundation CAREER Development award to A.R. Acknowledgment is also made to the donors of the Petroleum Research Fund, administered by the American Chemical Society. The authors are also grateful to Kevin Hallock and Katherine Henzler for useful discussions.

\section{REFERENCES}

1. Rothgeb TM, Oldfield E. J. Biol. Chem. 1981; 256: 6004

2. Seydel JK, Wassermann O. Biochem. Pharmacol. 1976; 25: 2357.

3. Römer J, Bickel MH. Biochem. Pharmacol. 1979; 28: 799.

4. Bickel MH, Steele JW. Chem. Biol. Interact. 1974; 8: 151.

5. Sanganahalli BG, Joshi PG, Joshi NB. Life Sci. 2000; 68: 81.

6. Hostetler KY, Matsuzawa Y. Biochem. Pharmacol. 1981; 30: 1121.

7. Albouz S, Lesaux F, Wenger D, Hauw JJ, Baumann N. Life Sci. 1986; 38: 357.

8. Albouz S, Vanier MT, Hauw JJ, Lesaux F, Boutry JM, Baumann N. Neurosci. Lett. 1983; 36: 311.

9. Albouz S, Tocque B, Hauw JJ, Boutry JM, Lesaux F, Bourdon R, Baumann N. Life Sci. 1982; 31: 2549.

10. Geist SH, Lullmannrauch R. Ann. Anat. 1994; 176: 3.

11. Xia Z, Ying G, Hansson AL, Karlsson H, Xie Y, Bergstrand A, DePierre JW, Nässberger L. Prog. Neurobiol. 2000; 60: 501.

12. Scuntaro I, Kientsch U, Wiesmann UN, Honegger UE. Br. J. Pharmacol. 1996; 119: 829.

13. Kucia K, Malecki A, Gabryel B, Trzeciak HI. Pol. J. Pharmacol. 2003; 55: 5.

14. Hurwitz R, Ferlinz K, Sandhoff K. Biol. Chem. Hoppe-Seyler 1994; 375: 447.

15. Arêas JA, Gröbner G, Glaubitz C, Watts A. Biochemistry 1998; 37: 5582.

16. Carbone MA, Macdonald PM. Biochemistry 1996; 35: 3368.

17. Mizushima T, Ishikawa Y, Obana E, Hase M, Kubota T, Katayama T, Kunitake T, Watanabe E, Sekimizu K. J. Biol. Chem. 1996; 271: 3633.
18. Wieprecht T, Apostolov O, Beyermann M, Seelig J. Biochemistry 2000; 39: 442.

19. Pasenkiewicz-Gierula M, Takaoka Y, Miyagawa H, Kitamura K, Kusumi A. Biophys. J. 1999; 76: 1228.

20. Pasenkiewicz-Gierula M, Róg T, Kitamura K, Kusumi A. Biophys. J. 2000; 78: 1376.

21. Hauser H, Pascher I, Pearson RH, Sundell S. Biochim. Biophys. Acta 1981; 650: 21.

22. Ho C, Slater SJ, Stubbs CD. Biochemistry 1995; 34: 6188.

23. Seelig J, Macdonald PM, Scherer PG. Biochemistry 1987; 26: 7535.

24. Ghosh R. Biochemistry 1988; 27: 7750.

25. Koga K, Kanazawa Y. Biochemistry 1980; 19: 2779.

26. Kanazawa Y, Koga K. Biochem. Biophys. Res. Commun. 1980; 95: 269.

27. Siminovitch DJ, Rance M, Jeffrey KR. FEBS Lett. 1980; 112: 79.

28. Siminovitch DJ, Jeffrey KR. Biochim. Biophys. Acta 1981; 645: 270.

29. Siminovitch DJ, Jeffrey KR, Eibl H. Biochim. Biophys. Acta 1983; 727: 122.

30. Siminovitch DJ, Brown MF, Jeffrey KR. Biochemistry 1984; 23: 2412.

31. Tamm LK, Seelig J. Biochemistry 1983; 22: 1474.

32. Scherer PG, Seelig J. Biochemistry 1989; 28: 7720.

33. Hallock KJ, Henzler Wildman K, Lee DK, Ramamoorthy A. Biophys. J. 2002; 82: 2499.

34. Washburn E, West CJ, Hull C. International Critical Tables of Numerical Data, Physics, Chemistry, and Technology. McGraw-Hill: New York, 1926.

35. Ulrich AS, Watts A. Biophys. J. 1994; 66: 1441.

36. Bechinger B, Seelig J. Chem. Phys. Lipids 1991; 58: 1.

37. Bennett AE, Rienstra CM, Auger M, Lakshmi KV, Griffin RGJ. Chem. Phys. 1995; 103: 6951.

38. Tattrie NH, Bennett JR, Cyr R. Can. J. Biochem. 1968; 46: 819.

39. Spuhler P, Anantharamaiah GM, Segrest JP, Seelig J. J. Biol. Chem. 1994; 269: 23904.

40. Bechinger B, Kim Y, Chirlian LE, Gesell J, Neumann JM, Montal M, Tomich J, Zasloff M, Opella SJ. J. Biomol. NMR 1991; 1: 167.

41. Koynova R, Caffrey M. Biochim. Biophys. Acta 1998; 1376: 91.

42. Habiger RGK, Seelig J. In The Structure and Conformation of Amphiphilic Membranes, Lipwsky R, Richter D, Kremer K (eds). Springer: Berlin, 1992; 49.

43. Green AL. J. Pharm. Pharmacol. 1967; 19: 10.

44. Meadows MD. In Chemistry. University of Illinois at UrbanaChampaign: Urbana, IL, 1979; 140.

45. Oldfield E, Adebodun F, Chung J, Montez B, Park KD, Le HB, Phillips B. Biochemistry 1991; 30: 11025.

46. Tabeta R, Mahajan S, Maeda M, Saito H. Chem. Pharm. Bull. (Tokyo) 1985; 33: 1793.

47. Casarotto MG, Craik DJ. J. Phys. Chem. 1992; 96: 3146.

48. Casarotto MG, Craik DJ. J. Phys. Chem. 1991; 95: 7093.

49. Santos JS, Lee DK, Hallock KJ, Ramamoorthy A. Recent Research Developments in Physical Chemistry, vol. 6 (part I). Transworld Research Network: Trivandrum, India, 2002; 179. 